\title{
Transcriptome analysis of colored calla lily (Zantedeschia rehmannii Engl.) by Illumina sequencing: de novo assembly, annotation and EST-SSR marker development
}

\author{
Zunzheng Wei ${ }^{1,2}$, Zhenzhen Sun ${ }^{3}$, Binbin Cui ${ }^{4}$, Qixiang Zhang ${ }^{1}$, Min Xiong ${ }^{2}$, Xian Wang ${ }^{2}$, Di Zhou ${ }^{\text {Corresp. }}{ }^{2}$ \\ 1 Beijing Key Laboratory of Ornamental Plants Germplasm Innovation \& Molecular Breeding, National Engineering Research Center for Floriculture and \\ College of Landscape Architecture, Beijing Forestry University, Beijing, China \\ 2 Key Laboratory of Biology and Genetic Improvement of Horticultural Crops (North China), Ministry of Agriculture, Key Laboratory of Urban Agriculture \\ (North), Ministry of Agriculture, Beijing Vegetable Research Center, Beijing Academy of Agriculture and Forestry Sciences, Beijing, China \\ 3 Beijing Key Laboratory of Separation and Analysis in Biomedicine and Pharmaceuticals, Beijing Institute of Technology, Beijing, China \\ 4 Department of Biology and Chemistry, Baoding University, Baoding, Hebei, China \\ Corresponding Author: Di Zhou \\ Email address: zhoudibvrc@163.com
}

Colored calla lily is the short name for the species or hybrids in section Aestivae of genus Zantedeschia. It is currently one of the most popular flower plants in the world due to its beautiful flower spathe and long postharvest life. However, little genomic information and few molecular markers are available for its genetic improvement. Here, de novo transcriptome sequencing was performed to produce large transcript sequences for $Z$. rehmannii cv. 'Rehmannii' using an Illumina HiSeq 2000 instrument. More than 59.9 million cDNA sequence reads were obtained and assembled into 39,298 unigenes with an average length of 1,038 bp. Among these, 21,077 unigenes showed significant similarity to protein sequences in the non-redundant protein database ( $\mathrm{Nr}$ ) and in the Swiss-Prot, Gene Ontology (GO), Cluster of Orthologous Group (COG) and Kyoto Encyclopedia of Genes and Genomes (KEGG) databases. Moreover, a total of 117 unique transcripts were then defined that might regulate the flower spathe development of colored calla lily. Additionally, 9,933 simple sequence repeats (SSRs) and 7,162 single nucleotide polymorphisms (SNPs) were identified as putative molecular markers. High-quality primers for 200 SSR loci were designed and selected, of which 58 amplified reproducible amplicons were polymorphic among 21 accessions of colored calla lily. The sequence information and molecular markers in the present study will provide valuable resources for genetic diversity analysis, germplasm characterization and marker-assisted selection in the genus Zantedeschia. 
1 Transcriptome analysis of colored calla lily (Zantedeschia rehmannii Engl.) by

2 Illumina sequencing: de novo assembly, annotation and EST-SSR marker

\section{3 development}

4 Zun-zheng Wei ${ }^{1,2, \dagger}$, Zhen-zhen Sun ${ }^{3, \dagger}$, Bin-bin Cui ${ }^{4}$, Qi-xiang Zhang ${ }^{1}$, Min Xiong ${ }^{2}$, Xian

5 Wang $^{2}$, Di Zhou ${ }^{2 *}$

$6 \quad{ }^{1}$ Beijing Key Laboratory of Ornamental Plants Germplasm Innovation \& Molecular

7 Breeding, National Engineering Research Center for Floriculture and College of

8 Landscape Architecture, Beijing Forestry University, Beijing100083, China;

$9 \quad{ }^{2}$ Key Laboratory of Biology and Genetic Improvement of Horticultural Crops (North

10 China), Ministry of Agriculture, Key Laboratory of Urban Agriculture (North), Ministry of

11 Agriculture, Beijing Vegetable Research Center, Beijing Academy of Agriculture and

12 Forestry Sciences, Beijing 100091, China;

$13{ }^{3}$ Beijing Key Laboratory of Separation and Analysis in Biomedicine and Pharmaceuticals,

14 Beijing Institute of Technology, Beijing 10081, China;

$15{ }^{4}$ Department of Biology and Chemistry, Baoding University, Baoding, Hebei 071000,

16 China;

$17 \dagger$ These authors contributed equally to this work.

\section{Corresponding Author:}

20 Di Zhou

21 Zhanghua Road No. 50, Haidian District, Beijing 100097, China

Email address: zhoudibvrc@163.com

\section{ABSTRACT}

Colored calla lily is the short name for the species or hybrids in section Aestivae of genus beautiful flower spathe and long postharvest life. However, little genomic information and few molecular markers are available for its genetic improvement. Here, de novo transcriptome sequencing was performed to produce large transcript sequences for $Z$. rehmannii cv. 'Rehmannii' using an Illumina HiSeq 2000 instrument. More than 59.9 million cDNA sequence reads were obtained and assembled into 39,298 unigenes with an average length of 1,038 bp. Among these, 21,077 unigenes showed significant similarity to protein sequences in the non-redundant protein database ( $\mathrm{Nr}$ ) and in the Swiss-Prot, Gene Ontology (GO), Cluster of Orthologous Group (COG) and Kyoto Encyclopedia of Genes and Genomes (KEGG) databases. Moreover, a total of 117 unique transcripts were then defined that might regulate the flower spathe development of colored calla lily. Additionally, 9,933 simple sequence repeats (SSRs) and 7,162 single nucleotide polymorphisms (SNPs) were identified as putative molecular markers. High-quality primers for 200 SSR loci were designed and selected, of which 58 amplified reproducible amplicons were polymorphic among 21 accessions of colored calla lily. The sequence information and molecular markers in the present study will provide valuable resources for genetic diversity analysis, germplasm characterization and marker-assisted selection in the genus Zantedeschia. 


\section{Manuscript to be reviewed}

44

\section{INTRODUCTION}

Zantedeschia species, commonly known as calla lily or arum lily, belong to the genus Zantedeschia in the family Araceae. These plants are native to central and southern Africa and generally grow in marshy places, on grassy slopes, and even at forest margins. The genus Zantedeschia is divided into two distinct sections (Letty, 1973; Singh, 1996): section Zantedeschia, with one evergreen species (Z. aethiopica Spreng.), and section Aestivae, with six deciduous species (Z. rehmannii Engl., Z. jucunda Letty., Z. elliottiana Engl., Z. pentlandii Wittm., Z. valida Singh. and Z. albomaculata Baill.). Z. aethiopica, also called white calla lily, is characterized by a pure white spathe and leaves that do not die down in the winter. In contrast, the species or hybrids of section Aestivae, also called colored calla lily, are characterized by a variety of spathe colors and have leaves that die down in the winter. In addition, a new species, Z. odorata Perry., that is dormant in the summer and has a white spathe that can produce an invariably delicate freesia-like scent, was classified into section Zantedeschia. There are a number of post-fertilization incompatibility barriers between the species in section Zantedeschia and section Aestivae, including endosperm degeneration, abnormal embryo development and arrested plastid development (Yao et al., 1994a; Yao et al., 1995).

As a famous flower plant, colored calla lily is very popular all over the world because of its beautiful flower spathe and long postharvest life. It is an important export flower crop for New Zealand, the Netherlands and the United States (Tjia, 1985; Funnell, 1993; Funnell \& MacKay, 1999). The extensive commercial production of colored calla lily for cut flowers and/or planting material occurs in Auckland and Palmerston North (New Zealand), South Holland (the Netherlands), California and Colombia (the United States) and so on. The potential application of colored calla lily for commercial tuber production, cut flowers, pot flowers and even as landscape specimens is also unlimited worldwide (Tjia, 1985). Significant advances have been made, especially for colored calla lily, through traditional breeding practices in the floriculture industry. A multitude of cultivars and hybrids showing great variation in flower color from dark red, pink, orange, yellow to even white are available at present. In fact, most of the cultivars that are hybridized, commercially developed, and used for cut or potted ornamental purposes belong to the species Z. rehmannii, Z. elliottiana, Z. pentlandii and Z. albomaculata (Funnell, 1993; Funnell \& MacKay, 1999).

Molecular markers are powerful genetic tools for gene mapping and molecular markerassisted selection (MAS) in the breeding of several crop species (Varshney et al., 2007). Previous studies on colored calla lily also have reported that several dominant markers, including random amplified polymorphic DNA (RAPD) and inter-simple sequence repeats (ISSR), were used for cultivar identification and to assess genetic diversity (Hamada \& Hagimori, 1996; Lu et al., 2012; Zhang et al., 2009; Chen et al., 2013; Lu et al., 2014). However, the current conventional breeding of calla lily in section Aestivae is still performed without the aid of molecular markers. It is perhaps the dominant inheritance pattern of these markers that hinders the detection of allelic information. In contrast, co- 


\section{Manuscript to be reviewed}

86 dominant markers, such as simple sequence repeats (SSRs) and single nucleotide

87 polymorphisms (SNPs), are the favored markers for the detection of allelic diversity.

88 Among these markers, SSR markers have gained attention due to their multi-allelic nature,

89 abundance throughout the genome, high reproducibility and polymorphism, adaptability to

90 automation and high-throughput genotyping (Morgante, Hanafey \& Powell, 2002).

91 Nevertheless, progress in developing SSR markers in section Aestivae and the genus

92 Zantedeschia is limited compared to that in other crop plants. Only one previous effort has

93 produced 43 polymorphic SSRs from 4,394 EST sequences of Z. aethiopica, representing

94 the first dataset of polymorphic EST-SSR markers for the genus Zantedeschia (Wei et al.,

95 2012). This conventional method of EST-SSR development has a high development cost

96 and a low throughput, which may restrict the use of SSR markers in further genetic

97 breeding (Morgante, Hanafey \& Powell, 2002; Varshney, Graner \& Sorrells, 2005).

98 Recent advances in RNA sequencing (RNA-seq) technology and de novo assembly

99 provide an excellent strategy for the efficient and cost-effective development of high-

100 throughput EST-SSRs. This strategy can enrich the large amounts of expressed sequence

101 data for non-model organisms for which the reference genome or transcriptome is not

102 available (Martin \& Wang, 2011). Several non-model organisms in the Araceae family,

103 including Z. aethiopica (Cândido et al., 2014), Arum concinnatum (Onda et al., 2015),

104 Anthurium andraeanum (Tian et al., 2013; Yang et al., 2015; Li et al., 2015), Pinellia

105 ternate (Wang et al., 2014), Amorphophallus konjac and A. bulbifer (Zheng et al., 2013;

106 Diao et al., 2014), have been recently studied by transcriptome sequencing, which has

107 provided a better understanding of these crops. EST-SSR markers have been successfully

108 characterized and developed in P. ternate (Wang et al., 2014), A. konjac and A. bulbifer

109 (Zheng et al., 2013). A total of 14,468 and 19,596 EST-SSRs were identified in 12,000 and

11016,027 non-redundant transcriptome unigenes, respectively, of $P$. ternate and two

111 Amorphophallus species (Wang et al., 2014; Zheng et al., 2013). Furthermore, 320 primers

112 were synthesized and used to validate the amplification and assessment of polymorphisms

113 in 25 individual Amorphophallus spp. plants (Zheng et al., 2013), of which 275 primer

114 pairs yielded PCR amplification products and 205 were polymorphic. This strongly

115 demonstrated that de novo assembly based on RNA-seq can offer a simple, direct and

116 reliable approach for the identification and development of massive unigene-based

117 microsatellite markers with diverse motifs. In addition to the development of molecular

118 markers, transcriptome sequencing has also been used for the discovery, profiling and

119 quantification of RNA transcripts and novel genes. For example, three lipid transfer

120 proteins (LTP) that are potentially involved in defense against pathogens or predators were

121 identified by the in silico screening of the 83,578 transcriptome contigs of $Z$. aethiopica

122 (Cândido et al., 2014). This represents the first transcriptome dataset for the genus

123 Zantedeschia.

124 Here, we presented the generation of a large expressed sequence dataset based on

125 Illumina HiSeq ${ }^{\text {TM }} 2000$ sequencing data from mixed tissues of colored calla lily, $Z$.

126 rehmannii cv. 'Rehmannii'. The objectives were as follows: (1) to characterize and

127 annotate the transcriptome information of colored calla lily; (2) to identify all of the 
128 candidate genes encoding enzymes or putative transcription factors that are involved in its

129 flower spathe development; (3) to analyze the frequency and distribution of SSRs and

130 SNPs in its transcribed regions; (4) to develop a large set of EST-SSR markers; and (5) to

131 evaluate the transferability and genetic diversity of 21 cultivars or hybrids of colored calla

132 lily using these EST-SSR markers.

133

\section{MATERIALS AND METHODS}

\section{Plant materials}

136 All of the samples used in the present study were maintained at the Bulb and Perennial

137 Flowers Genebank (BPFG), Beijing Academy of Agriculture and Forestry Sciences,

138 Beijing, China. The samples were grown in the experimental greenhouse in Yanqing Farm

139 (latitude $40.46 \mathrm{~N}$, longitude 115.91E); this cultivation did not involve endangered or

140 protected species. Z. rehmannii cv. Rehmannii, a potted cultivar with a pink spathe, was

141 selected for transcriptome sequencing. An additional twenty accessions (Table S1),

142 including nine individuals from New Zealand (Elmaro, Pink Diamond, Butter Gold, Black

143 Magic, Majestic Red, Sunny Baby, Greta, Goldilocks and Gold affair), five from the

144 Netherlands (Odessa, Captain Reno, Allure, Captain Romance, and Captain Murano), three

145 from the United States (Parfait, Super Gem and Rose Gem) and three from China (Jingcai

146 Yangguang, $\mathrm{ZH}$ and Xiangyuan Red), were used to estimate microsatellite variations of the

147 EST-SSR markers developed in our study. All of the accessions are released commercial

148 varieties of New Zealand, the Netherlands, the United States or China; thus, permission for

149 collection is not required.

150 RNA extraction, cDNA library construction and Illumina sequencing

151 To achieve a comprehensive collection of expressed transcripts of colored calla lily, ten

152 'Rehmannii' tissue types, including root, tuber, stem, leaf, bud, spathe, pistillate

153 inflorescence and staminate inflorescence, immature seed and mature seed, were separately

154 harvested at the anthesis and wilting stages. All of the tissue samples were immediately

155 frozen in liquid nitrogen and stored at $-80^{\circ} \mathrm{C}$ until RNA extraction. Total RNA Trizol

156 Reagent (Invitrogen, USA) was used to extract RNA from all of the samples, following the

157 manufacturer's instructions. The RNA purity and concentration were determined using a

158 NanoDrop spectrophotometer (Thermo, USA). Equal quantities of total RNA from each

159 sample were then pooled together and used for transcriptome sequencing.

160 cDNA library construction and Illumina-based sequencing were performed by the

161 Shanghai Majorbio Bio-pharm Biotechnology Co. Ltd. (Shanghai, China) according to the

162 manufacturer's instructions. The paired-end cDNA sequencing libraries with approximate

163 average insert lengths of 200 base pairs were prepared from the total RNA, as per the

164 protocol of the Genomic Sample Prep kit (Illumina, San Diego, CA). This process started

165 with mRNA fragmentation, followed by reverse transcription, first- and second-strand

166 synthesis, paired-end adapter ligation and PCR amplification. Library quantification and

167 quality assessment were performed on an Agilent 2100 Bioanalyzer and an ABI

168 StepOnePlus Real-Time PCR System. Finally, the cDNA library was sequenced as 101-

169 mer $\times 2$ on an Illumina HiSeq 2000 using paired-end sequencing chemistry. 
170 De novo transcriptome assembly and functional annotation

171 The raw sequencing image data were transformed into raw reads and stored in FASTQ

172 format. These raw data were then filtered and deposited in the National Center for

173 Biotechnology (NCBI) Sequence Read Archive (SRA) under accession number

174 SRR3310941. All of the reads with adaptor contamination, empty reads, non-coding RNA

175 (such as rRNA, tRNA and miRNA), ambiguous nucleotides comprising more than 5\% or

176 low quality value (QV), i.e., an average $\mathrm{QV}$ of less than $20(\mathrm{QV}<20)$, were discarded or

177 filtered. Then, the clean and high-quality transcriptome sequence data were de novo

178 assembled using the short reads assembling program Trinity (Haas et al., 2013) with

179 default settings. An assembled transcripts database (File S2) was finally achieved and

180 arranged according to the gene family clustering analysis. These final assembled

181 transcripts were used for further bioinformatics analysis.

182 The assembled sequences were functionally annotated by BLASTx against a series of

183 databases, including the Nr, Swiss-Prot, COG, and KEGG databases, with a common

184 significance threshold cut-off of E-value $1 \mathrm{e}-10^{-5}$. Based on $\mathrm{Nr}$ annotation, Blast2GO

185 (https://www.blast2go.com/) and WEGO (http://wego.genomics.org.cn/cgi-bin/wegol)

186 software were used to retrieve and classify GO annotation categories defined by molecular

187 function, cellular component and biological process.

188 Marker locus detection and SSR primer pair design

189 MIcroSAtellite identification tool (MISA, http://pgrc.ipk-gatersleben.de/misa/misa.html)

190 was used to identify microsatellites in the assembled transcripts. The minimum number of

191 repeats used to select the SSRs was ten for mononucleotide repeats, six for dinucleotide

192 repeats, and five for tri-, tetra-, penta-, and hexanucleotide repeats. Primer Premier 5.0

193 (PREMIER Biosoft International, Palo Alto, CA) was then used to manually design 200

194 pairs of PCR primers to randomly select sequences with SSR loci. The design criteria of

195 the primers were as follows: primer length 18-24 bp; GC content 40-65\%; melting

196 temperature $50-65^{\circ} \mathrm{C}$; and expected product size $100-300 \mathrm{bp}$ with no secondary structures.

197 All 200 primer pairs were synthesized by Sangon Biological Engineering Technology

198 (Shanghai, China).

199 Potential SNPs were detected using the programs BWA (http://biobwa.sourceforge.net/)

200 and VarScan (http://varscan.sourceforge.net/). The assembled unigenes were used as

201 references to BLAST the raw sequencing reads.

202 DNA extraction and EST-SSR marker amplification

203 The young leaves were collected from 21 accessions (Table S1) grown at BPFG. The total

204 genomic DNA was extracted using the DNeasy Plant Mini Kit (Zexing Biotech, China)

205 following the manufacturer's protocol. The quantity and quality of DNA were evaluated

206 using a Nanodrop ND 1000 spectrophotometer (Thermo Scientific, USA). The DNA was

207 adjusted to a concentration of $20 \mathrm{ng} / \mu \mathrm{l}$ and stored at $-20^{\circ} \mathrm{C}$ until use.

208 The SSR amplification reactions were conducted as described previously by Wei et al.

209 (2012) with little modification. The PCR reaction mixtures $(10 \mu \mathrm{l})$ contained $1 \times$ PCR

210 buffer, 20-30 ng of template DNA, $0.8 \mathrm{mM} \mathrm{MgCl}_{2}, 15 \mu \mathrm{M}$ dNTPs, $0.25 \mu \mathrm{M}$ each primer,

211 and $0.2 \mathrm{U}$ of Taq DNA polymerase (Zexing Biotech, China). PCR amplifications were 
212 performed in the GeneAmp PCR System 9700 (Applied Biosystems). The thermal profile

213 included an initial denaturation at $95^{\circ} \mathrm{C}$ for $5 \mathrm{~min}$, followed by 20 cycles of $95^{\circ} \mathrm{C}$ for $30 \mathrm{~s}$,

$21450-60^{\circ} \mathrm{C}$ for $45 \mathrm{~s}$, and $72^{\circ} \mathrm{C}$ for $60 \mathrm{~s}$ and a final extension at $72^{\circ} \mathrm{C}$ for $10 \mathrm{~min}$. The PCR

215 products were separated on $8.0 \%$ polyacrylamide non-denaturing gels and visualized by

216 silver staining (Wei et al., 2012). The product sizes were determined by comparison with a

217 34- to 501-bp pUC19/MspI DNA marker (Zexing Biotech, China)

218 Data analysis

219 The number of alleles $(\mathrm{Na})$, observed heterozygosity $(\mathrm{Ho})$, expected heterozygosity $(\mathrm{He})$,

220 and polymorphic information content (PIC) were calculated using GenAlEx 6.4 (Peakall \&

221 Smous, 2006) and Power Marker Version 3.25 (Liu \& Muse, 2005). A cluster analysis was

222 conducted and displayed using the neighbor-joining (NJ) algorithm as implemented in

223 Power Marker Version 3.25 (Liu \& Muse, 2005) and MEGA version 5.0 (Tamura et al.,

224 2011).

225

226 RESUTLTS

227 Illumina sequencing and de novo assembly

228

229 Table 1 Summary of transcriptome statistics and functional annotation for colored calla lily

230 'Rehmannii'

\begin{tabular}{lll}
\hline & Number & Percentage \\
\hline Raw reads & $59,882,890$ & \\
Total sizes (nt) & $6,048,171,890$ & \\
Clean reads & $46,343,613$ & \\
Transcripts & 62,382 & \\
Unigenes & 39,298 & \\
Unigenes (300-500 nt) & 13,367 & $34.02 \%$ \\
Unigenes (500-1,000 nt) & 13,088 & $33.30 \%$ \\
Unigenes (1,000-1,500 nt) & 4,975 & $12.65 \%$ \\
Unigenes (1,500-2,000 nt) & 2,988 & $7.60 \%$ \\
Unigenes (> 2,000 nt) & 4,880 & $12.41 \%$ \\
Mean length (nt) & 1,038 & \\
N50 (nt) & 1,476 & \\
GC\% & $45.74 \%$ & $53.51 \%$ \\
Annotated in Nr & 21,029 & $43.03 \%$ \\
Annotated in Swiss-Prot & 16,908 & $17.13 \%$ \\
Annotated in COG & 6,731 & $39.57 \%$ \\
Annotated in GO & 15,552 & $11.53 \%$ \\
Annotated in KEGG & 4,532 & $53.63 \%$ \\
Annotated in at least one database & 21,077 & $100 \%$ \\
Total unigenes & 39,298 & \\
\hline
\end{tabular}

231 
232 To provide a comprehensive transcriptome database for calla lily, a mixed cDNA library of

233 cultivar 'Rehmannii' from ten sampled tissues, i.e., root, tuber, stem, leaf, bud, spathe,

234 pistillate inflorescence and staminate inflorescence, immature seed and mature seed, was

235 constructed and sequenced using Illumina paired-end technology. A summary of the

236 sequencing output statistics is shown in Table 1. The sequencing yielded approximately

23759.9 million raw reads with a total of more than 6.1 billion nucleotides. After removing

238 ambiguous, low-quality reads and reads with adaptors, 46.3 clean reads were obtained for

239 further assembly. The de novo assembly yielded 62,382 transcripts and 39,298 unigenes.

240 The sequences of all the assembled unigenes were provided in FASTA format in Table S2.

241 These sequences contained $40.8 \mathrm{Mb}$ of sequence with an average size of 1,038 bp and an

242 N50 of 1,476 bp. The lengths of the unigenes ranged from 351 to $15,521 \mathrm{bp}$. Of these

243 unigenes, $67.3 \%(26,455)$ were shorter than $1,000 \mathrm{bp}, 20.3 \%(7,963)$ ranged from 1,000 to

$2442,000 \mathrm{bp}$, and the remaining $12.4 \%(4,880)$ were longer than 2,000 bp (Table 1). These

245 unigenes formed a potential pool for identification of genes and functional molecular

246 markers in colored calla lily.

247 Functional annotation of the unigenes

248

A

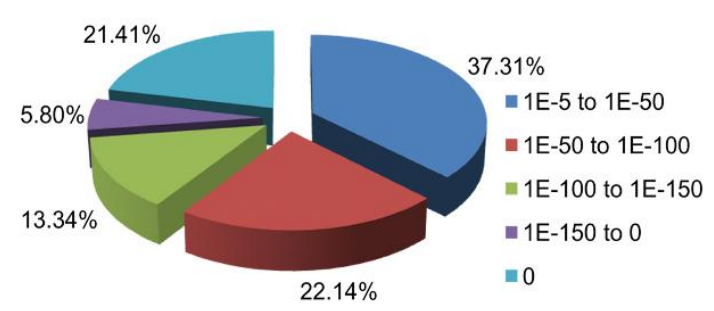

B

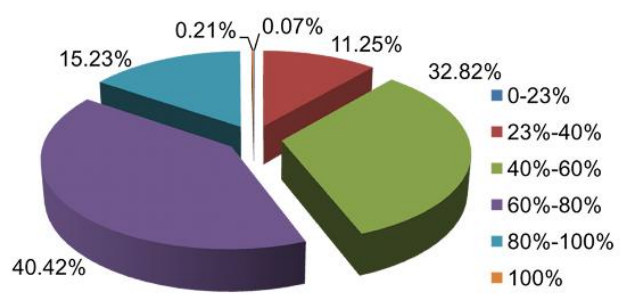

C

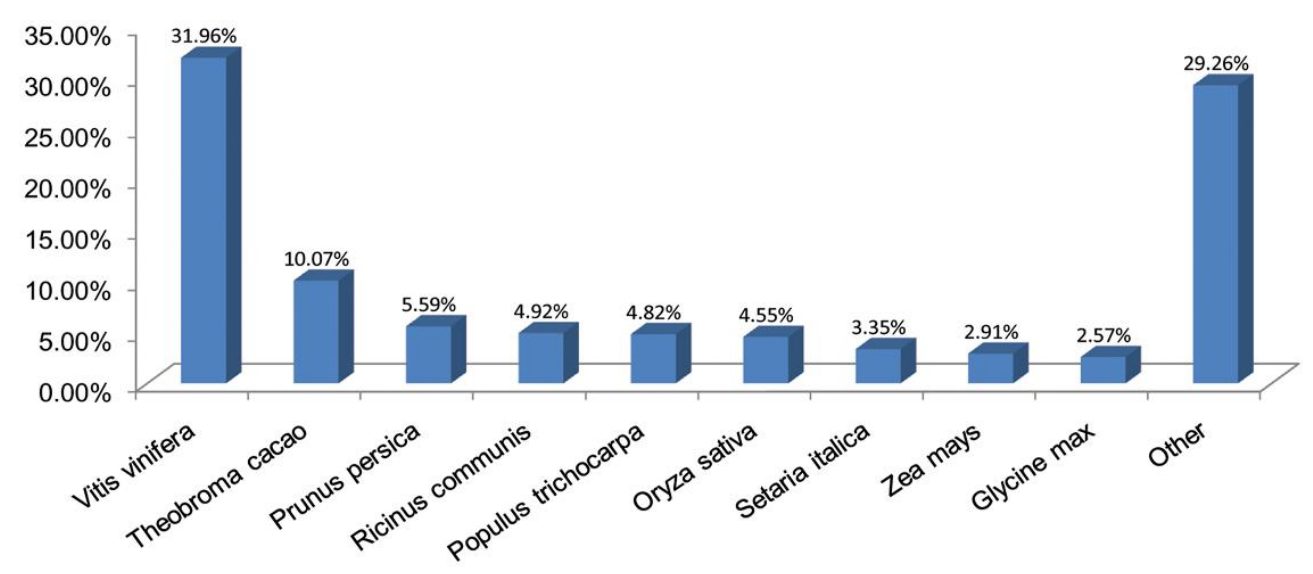

250 Figure 1 Characteristics of homology search for colored calla lily 'Rehmannii' unigenes 251 against non-redundant protein database $(\mathrm{Nr})$ with an E-Value $\leq 1 \mathrm{E}-10^{-5}$. (A) The E-value

252 distribution of BLASTx hit for each assembled unigene; (B) The similarity distribution of

253 BLASTx hits for each assembled unigenes; (C) Species-based distribution of the top

254 BLASTx hits for each assembled unigenes. 
256 Functional annotations were performed by a homology-based approach for cultivars

257 'Rehmannii' assembled transcripts. A sequence similarity search was first conducted

258 against the $\mathrm{Nr}$ and Swiss-Prot databases using the BLASTx algorithm with an E-value

259 threshold of $10^{-5}$ (Table 1 and Table S3). As shown in Table 1, the results indicated that

$26021,029(53.5 \%)$ of the 39,298 unigenes showed significant BLASTx matches in the $\mathrm{Nr}$

261 database, while 16,908 (43.0\%) were similar to proteins in the Swiss-Prot database. The

262 proportion of sequences showing hits in both the $\mathrm{Nr}$ and Swiss-Prot databases was higher

263 among the longer assembled transcripts. More than $55.0 \%$ of the unigenes longer than

2641,000 bp showed homologous matches while fewer than $45.0 \%$ of the unigenes shorter

265 than 1,000 bp showed matches (File S2). The E-value, sequence similarity and species

266 distributions of the top hits in the $\mathrm{Nr}$ database were also analyzed. The E-value distribution

267 of the top hits showed that $62.7 \%$ of the annotated sequences had high homology with E-

268 value $<10^{-50}$, whereas $37.3 \%$ showed a moderate homology with E-values from $10^{-5}$ to $10^{-}$

$2695^{50}$ (Fig. 1A). For the sequence similarity distribution analysis, 2,365 (11.3\%), 6,902

270 (32.8\%), 8,500 (40.4\%), 3,202 (15.2\%) and 45 (0.21\%) sequences were 23-40\%, 40-60\%,

$27160-80 \%, 80-100 \%$ and $100 \%$ similar in the $\mathrm{Nr}$ database, respectively (Fig. 1B). In addition,

272 the species distribution showed that Vitis vinifera (Vitaceae) was ranked first, with 6,720

273 (32.0\%) top BLASTx hits, followed by Theobroma cacao (Sterculiaceae), Prunus persica

274 (Rosaceae), Ricinus communis (Euphorbiaceae) and Populus trichocarpa (Salicaceae),

275 with 2,117 (10.1\%), 1,176 (5.6\%), 1,034 (4.9\%), and 1,014 (4.8\%) hits, respectively (Fig.

276 1C).

277 Functional classification of the unigenes

278 
A

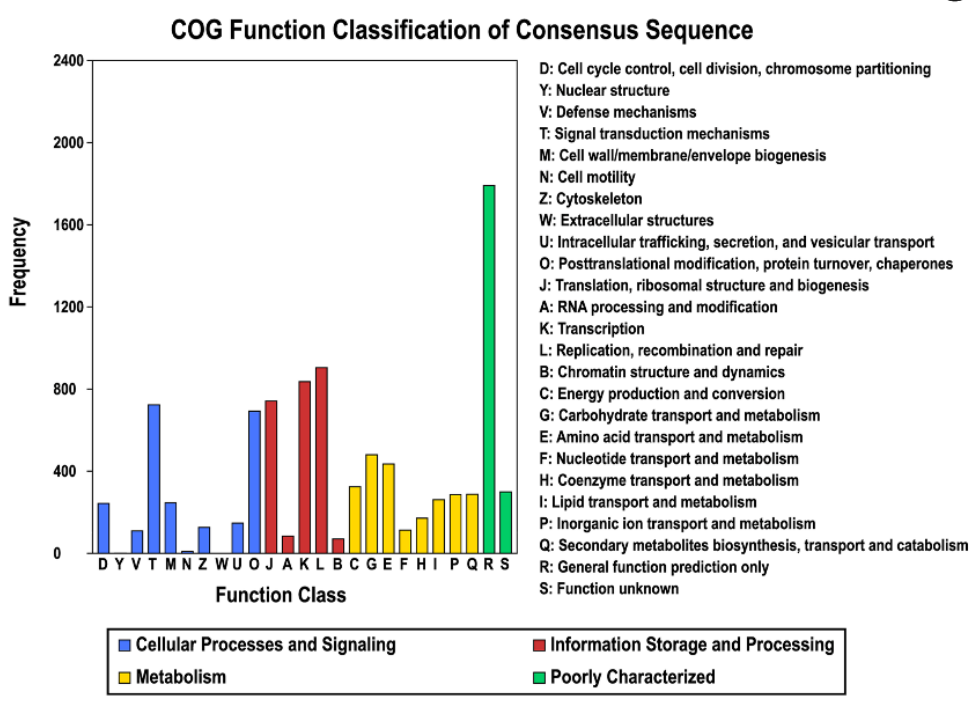

B

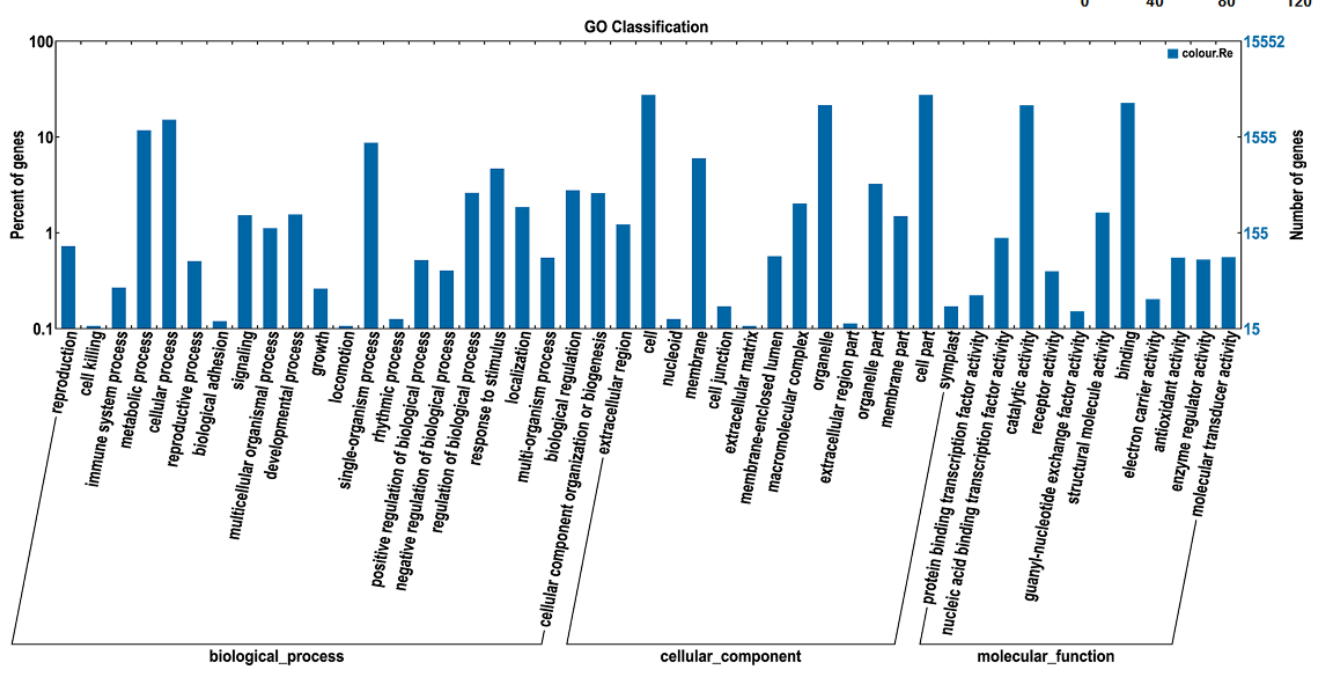

C

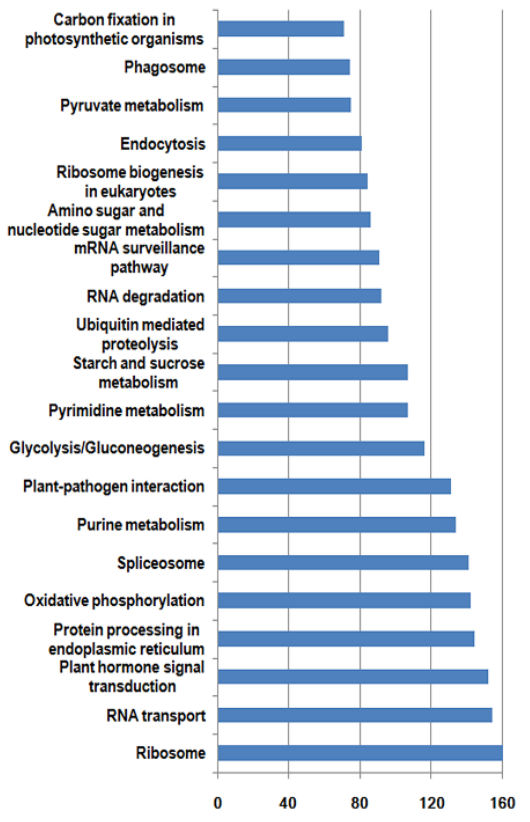
(n) 
295 genomes of bacteria, archaea and eukaryotes (Roman et al., 2000). The COG function

296 classification of the 'Rehmannii' sequences is shown in Fig. 2A. In total, 6,731 of the

29739,298 unigenes showing Nr hits were functionally annotated and classified into 24 COG

298 categories, including cellular structure, biochemistry metabolism, molecular processing,

299 and signal transduction, among others. Given that 9,379 COG-annotated putative proteins

300 were obtained, some of these unigenes were assigned to multiple COG classifications. The

301 cluster for general function prediction only $(1,791,26.6 \%)$ represented the largest group,

302 followed by replication, recombination and repair $(904,13.4 \%)$ and transcription $(836$,

$30312.4 \%)$. Additionally, only a few unigenes were assigned to cell motility $(9,0.13 \%)$ and

304 nuclear structure $(1,0.01 \%)$.

305 The GO database, an internationally standardized gene functional classification system,

306 offers dynamic and updated gene ontology that defines gene products in terms of their

307 associated cellular component, molecular function, and biological process in any organism

308 (Ashburner et al., 2007). Based on $\mathrm{Nr}$ annotation, 15,552 (39.6\%), unigenes in the present

309 study were assigned to GO classes with 15,964 functional terms. A summary of the

310 'Rehmannii' unigenes classified to each GO Slim term is shown in Fig. 2B. The annotated

311 gene sequences that belong to the cellular component, molecular function, and biological

312 process categories were divided into 47 functional groups. Under the cellular component

313 category, cell $(4,254,27.4 \%)$ and cell part $(4,253,27.4 \%)$ were the most highly represented

314 group, followed by organelle $(3,331,21.4 \%)$. For the molecular function category, the top

315 two groups were binding $(3,512,22.6 \%)$ and catalytic activity $(3,313,21.3 \%)$. However,

316 the majority of the groups, including antioxidant activity, receptor activity, protein binding

317 transcription factor activity, nutrient reservoir activity, etc., contained only a few unigenes

318 (655, 4.2\%). For biological processes, the majority of the unigenes were involved in

319 cellular processes $(2,344,15.1 \%)$ and metabolic processes $(1,814,11.7 \%)$, indicating that

320 important metabolic and cellular activities occur in 'Rehmannii'. Genes involved in other

321 important biological processes, such as single-organism processes $(1,337,8.6 \%)$, stimulus

322 response $(712,4.6 \%)$ and biological regulation $(416,2.7 \%)$, also accounted for a large

323 fraction of the annotated transcripts. Furthermore, we found that a portion of the unigenes

324 were assigned to developmental processes $(226,1.5 \%)$, reproductive processes $(63,0.4 \%)$

325 and growth $(25,0.2 \%)$ and may be involved in flower-related biological processes, such as

326 flower-type development and formation, in colored calla lily.

327 The KEGG database (Kanehisa et al., 2004) determines the biological pathways in

328 which protein and small-molecule interactions occur. A total of 4,532 assembled sequences

$329(11.5 \%)$ were consequently annotated and assigned to 117 predicted metabolic pathways.

330 The number of sequences in each pathway ranged from 1 to 160 . Fig. $2 \mathrm{C}$ shows the top 20

331 KEGG metabolic pathways represented by unique transcripts of calla lily. Ribosome

332 represented the largest number of transcript sequences in our dataset (160), followed by

333 RNA transport (154) and plant hormone signal transduction (152). Among the annotated

334 sequences, more than one-third $(1,661)$ were classified into 46 metabolic groups, such as

335 purine metabolism (134), pyrimidine metabolism (107), starch and sucrose metabolism 
336 (75), and so on. This indicates that diverse metabolic processes are active and a variety of

337 metabolites are synthesized in the tissues and organs of colored calla lily.

338 Overall, 21,077 unique sequence-based annotations using the selected Nr, Swiss-Prot,

339 COG, GO and KEGG databases were assigned to assembled transcripts of the cultivar

340 'Rehmannii'. The functional analysis revealed that it is feasible to obtain transcriptome

341 sequences through high-throughput technology, even for non-model plants with large

342 genomes. Furthermore, all of these functional annotation assignments provide valuable

343 information for colored calla lily to investigate specific biochemical and developmental

344 processes and potential gene structures, functions and pathways.

\section{Unigenes related to flower development in colored calla lily}

346 Enzymes functioning in flower development have been well documented in many plants.

347 Based on $\mathrm{Nr}$ annotation results of colored calla lily unigenes, we identified a total of 171

348 candidate transcripts encoding enzymes related to pigment biosynthesis (61), floral organ

349 development (26), flowering regulation (63) and flower senescence (31). The EST

350 sequences of all these unigenes are listed in Table S4. Flower development is a complex

351 process controlled by an integrated network of multi-genetic pathways in higher plants.

352 The putative gene homologs identified in the present study were involved in eight

353 pathways, including the anthocyanin biosynthesis pathway (52), carotenoid biosynthesis

354 pathway (9), specification of floral organ identity (26), photoperiod pathway (22),

355 vernalization pathway (11), gibberellic acid pathway (11), autonomous or other pathways

356 (9) and ethylene biosynthesis pathway (31). The identification and analysis of these key

357 genes will provide a foundation for understanding the potential molecular genetic

358 mechanisms controlling different aspects of floral development of colored calla lily in the

359 future.

360 SSR and SNP loci discovery

361

362 Table 2 Features of the SSR repeat types identified in colored calla lily 'Rehmannii'

363 unigenes.

\begin{tabular}{ll}
\hline Feature & Colored calla lily \\
\hline Total number of sequences examined & 39,298 \\
Total size of examined sequences $(\mathrm{Mb})$ & 40.78 \\
Total number of identified SSRs & 9,933 \\
Number of SSR-containing sequences & 7,997 \\
Number of sequences containing more than one SSR locus & 1,556 \\
Number of SSRs present in compound formation & 580 \\
\hline
\end{tabular}

365 Transcriptome sequencing is important for identification and development of molecular

366 markers, such as SSRs and SNPs. For the development of new markers for colored calla

367 lily, all of the 39,298 unigenes generated in this study were used to mine potential

368 microsatellites using MISA software. A total of 9,933 potential EST-SSRs were identified

369 in 7,997 unigenes, of which 1,556 sequences contained more than one SSR, and 580 SSRs

370 were present in compound form (Table 2 and Table S5). Considering that approximately 
$37140,780 \mathrm{~kb}$ was analyzed, we detected a frequency of at least one SSR per $4.1 \mathrm{~kb}$ in the

372 expressed fraction of the 'Rehmannii' genome.

373

374 Table 3 Summary of EST-SSRs identified from the unigenes of colored calla lily

375 'Rehmannii'.

\begin{tabular}{|c|c|c|c|c|c|c|c|c|}
\hline \multirow{2}{*}{ Repeat motif } & \multicolumn{8}{|c|}{ Number of repeats } \\
\hline & 5 & 6 & 7 & 8 & 9 & 10 & $>10$ & Total \\
\hline \multicolumn{9}{|c|}{ Di- $(3,482, \quad 59.78 \%)$} \\
\hline $\mathrm{AG} / \mathrm{CT}$ & 0 & 652 & 568 & 525 & 536 & 381 & 106 & 2,768 \\
\hline $\mathrm{AT} / \mathrm{AT}$ & 0 & 108 & 68 & 61 & 46 & 49 & 28 & 360 \\
\hline $\mathrm{AC} / \mathrm{GT}$ & 0 & 132 & 70 & 54 & 25 & 32 & 15 & 328 \\
\hline $\mathrm{CG} / \mathrm{CG}$ & 0 & 14 & 7 & 0 & 3 & 2 & 0 & 26 \\
\hline \multicolumn{9}{|c|}{ Tri- $(2,261,38.82 \%)$} \\
\hline $\mathrm{AGG} / \mathrm{CCT}$ & 316 & 116 & 43 & 4 & 1 & 0 & 0 & 480 \\
\hline AAG/CTT & 258 & 136 & 67 & 6 & 0 & 0 & 1 & 468 \\
\hline $\mathrm{AGC} / \mathrm{CTG}$ & 252 & 99 & 39 & 2 & 0 & 0 & 0 & 392 \\
\hline $\mathrm{CCG} / \mathrm{CGG}$ & 245 & 85 & 39 & 3 & 0 & 0 & 0 & 372 \\
\hline ATC/ATG & 96 & 37 & 21 & 3 & 0 & 0 & 0 & 157 \\
\hline ACC/GGT & 84 & 42 & 20 & 3 & 1 & 0 & 0 & 150 \\
\hline Other & 146 & 49 & 35 & 11 & 1 & 0 & 0 & 242 \\
\hline \multicolumn{9}{|l|}{ Tetra- $(62,1.06 \%)$} \\
\hline AAAG/CTTT & 14 & 2 & 0 & 0 & 0 & 0 & 0 & 16 \\
\hline AGAT/ATCT & 12 & 1 & 0 & 0 & 0 & 0 & 0 & 13 \\
\hline ACAT/ATGT & 4 & 1 & 1 & 0 & 0 & 0 & 0 & 6 \\
\hline AAAT/ATTT & 5 & 0 & 0 & 0 & 0 & 0 & 0 & 5 \\
\hline Others & 17 & 3 & 1 & 1 & 0 & 0 & 0 & 22 \\
\hline Penta- $(13,0.22 \%)$ & 13 & 0 & 0 & 0 & 0 & 0 & 0 & 13 \\
\hline Hexa- $(7,0.12 \%)$ & 3 & 0 & 2 & 1 & 0 & 0 & 1 & 7 \\
\hline Total & 1465 & 1477 & 981 & 674 & 613 & 464 & 151 & 5825 \\
\hline Percentage & $25.15 \%$ & $25.36 \%$ & $16.84 \%$ & $11.57 \%$ & $10.52 \%$ & $7.97 \%$ & $2.59 \%$ & $100 \%$ \\
\hline
\end{tabular}

376

377

378

379

380

381

382

383

384

385

386

The type and frequency of EST-SSRs with different numbers of tandem repeats are summarized in Table 3. Because mononucleotide repeats may not be accurate due to sequencing errors and assembly mistakes, they were excluded from further analyses.

Results showed that the identified SSR type was not evenly distributed throughout all the SSR-containing sequences of 'Rehmannii'. The dinucleotide repeat motifs were the most abundant $(3,482$ or $59.8 \%)$, followed by trinucleotide repeat motifs $(2,261$ or $38.8 \%)$, whereas hexa- (62 or $1.06 \%)$, penta- (13 or $0.22 \%)$ and tetranucleotide repeat motifs ( 7 or $0.12 \%)$ were rare. The number of SSR repeats ranged from 5 to 24 , with 6 repeats $(1,477$, $25.4 \%)$ being the most common, followed by 5 tandem repeats $(1,465,25.2 \%)$ and 7 tandem repeats $(981,16.8 \%)$. Motifs containing more than 10 repeats were rare (Table S3), 
387 with a frequency of only $2.6 \%$ (151). Within these SSRs, 47 motif sequence types were

388 identified, of which di-, tri-, tetra-, penta-, and hexanucleotide repeats had 4, 10, 13, 13 and

3897 types, respectively. AG/CT alone accounted for $79.5 \%(2,768)$ of the total dinucleotide

390 repeats, followed by AT/TA $(360,10.4 \%)$ and AC/GT $(328,9.4 \%)$. Among the

391 trinucleotide repeats, GAA/CTT and AAG/CTT were the most abundant $(480,21.2 \%$; 468,

392 20.7\%). Other repeats, AGC/CTG, CCG/CGG, ATC/ATG and ACC/GGT, constituted

$39347.4 \%$ of the trinucleotide repeats.

394

395 Table 4 Summary of SNPs identified from unigenes of colored calla lily 'Rehmannii'.

\begin{tabular}{lllll}
\hline Transitions & Number & Transversions & Number & 396 \\
\hline $\mathrm{C} / \mathrm{T}$ & 2,262 & $\mathrm{~A} / \mathrm{T}$ & 650 & \\
$\mathrm{~A} / \mathrm{G}$ & 2,188 & $\mathrm{~A} / \mathrm{C}$ & 647 \\
& & $\mathrm{~T} / \mathrm{G}$ & 625 \\
& & $\mathrm{C} / \mathrm{G}$ & 790 \\
Total & 4,450 & Total & 2,712 \\
\hline
\end{tabular}

397

398

399

400

401

402

403

404

405

406

407

408

409

410

411

412

413

414

415

416

417

418

In addition to EST-SSRs, a total of 7,162 potential high-quality SNPs were identified by mapping against 39,298 reference unigenes. The overall frequency of all types of SNPs was one SNP per $5.69 \mathrm{~kb}$. The predicted SNPs included 4,450 transitions and 2,712 transversions (Table 4). The most abundant SNPs detected were C/T $(2,262,31.6 \%)$, followed by A/G $(2,188,30.6 \%)$ and $\mathrm{C} / \mathrm{G}(790,11.0 \%)$. The numbers of the remaining three SNP types (A/T, A/C, and T/G) were similar, each accounting for less than $10 \%$. Potential SNPs are shown in Table S6.

\section{SSR primer design, polymorphism detection and phylogenetic analysis}

A total of 200 EST-SSR loci (repeat motif >1) with appropriate flanking sequences were randomly selected for the design and synthesis of PCR primer pairs. Detailed information of the EST-SSR markers is shown in Table S7. A germplasm panel of five colored calla lily accessions (Rehmannii, Super Gem, Rose Gem, Xiangyuan Red and Allure) was initially used to validate the usefulness of EST-SSR markers in monitoring polymorphisms. A total of $137(68.5 \%)$ of the primer pairs were successfully amplified by PCR, while the remaining failed to generate any clear DNA products. Of the working primer pairs, 77 (56.2\%) produced clear PCR amplicons of the expected sizes, whereas 60 (43.8\%) amplified non-specific products, of which 23 markers generated PCR products larger or smaller than expected and 37 generated more than one band (Table S5).

Table 5 Characteristics of the 58 polymorphic EST-SSR markers in 21 colored calla lily accessions.

\begin{tabular}{llllll|llllll}
\hline Locus & Na & Ne & Ho & He & PIC & Locus & Na & Ne & Ho & He & PIC \\
\hline CallaRe015 & 3 & 2.057 & 0.684 & 0.514 & 0.425 & CallaRe110 & 2 & 1.324 & 0.286 & 0.245 & 0.215 \\
CallaRe016 & 6 & 3.320 & 0.600 & 0.699 & 0.654 & CallaRe117 & 3 & 2.410 & 0.000 & 0.585 & 0.513 \\
CallaRe028 & 4 & 3.756 & 1.000 & 0.734 & 0.685 & CallaRe118 & 4 & 2.028 & 0.667 & 0.507 & 0.462 \\
CallaRe030 & 3 & 2.095 & 1.000 & 0.523 & 0.409 & CallaRe120 & 4 & 2.932 & 0.333 & 0.659 & 0.593 \\
\hline
\end{tabular}




\begin{tabular}{|c|c|c|c|c|c|c|c|c|c|c|c|}
\hline 31 & 2 & 1.446 & 0.286 & 0.308 & 0.261 & CallaRe128 & 4 & 3.556 & 0.550 & 0.719 & 0.670 \\
\hline CallaRe032 & 2 & 930 & 810 & 482 & & & 2 & .984 & 0.545 & 496 & .373 \\
\hline & 2 & & & 229 & & 31 & & .930 & .286 & 659 & 0.601 \\
\hline & 4 & & & & & & 2 & & & & \\
\hline & 3 & & & & & 44 & 4 & 2.766 & 0.588 & 0.638 & 0.589 \\
\hline & 2 & & & & & 46 & 3 & 2.085 & 0.952 & .520 & 0.408 \\
\hline 049 & 2 & 960 & & 0.490 & & 147 & 3 & .841 & 0.353 & 0.457 & 0.411 \\
\hline allaRe050 & 6 & 3.630 & 0.571 & 0.724 & 0.68 & allaPe151 & 3 & 2.455 & 0.667 & 0.593 & 0.505 \\
\hline CallaRe055 & 3 & 2.057 & 0.526 & 0.514 & & CallaRe155 & 2 & 1.724 & 0.000 & 0.420 & 0.332 \\
\hline CallaRe056 & 2 & 1.946 & 0.833 & 0.486 & 0.368 & 156 & 4 & 2.309 & 0.667 & 0.567 & 0.486 \\
\hline CallaRe061 & 4 & 3.469 & 0.286 & 0.712 & 0.661 & CallaRe160 & 2 & 1.893 & 0.000 & 0.472 & 0.360 \\
\hline CallaRe066 & 2 & 1.265 & 0.238 & 0.210 & 0.1 & CallaRe165 & 2 & 2.000 & 1.000 & 0.412 & 0.375 \\
\hline CallaRe075 & 2 & 1.358 & 0.313 & 0.264 & 0.2 & CallaRe166 & 4 & 2.520 & 0.333 & 0.603 & 0.541 \\
\hline CallaRe078 & 4 & 2.303 & .952 & 0.566 & 0.471 & CallaRe170 & 3 & 2.597 & 0.737 & 0.615 & 0.536 \\
\hline CallaRe080 & 4 & 2.285 & 0.381 & 0.562 & 0.519 & CallaRe175 & 6 & 4.762 & 0.800 & 0.790 & 0.757 \\
\hline 081 & 3 & & 333 & 289 & & allat & 2 & 1.835 & 0.700 & 0.455 & 0.351 \\
\hline & 3 & & & & & 19 & 2 & 1.992 & & 498 & 0.374 \\
\hline & 2 & & & & & & 3 & & & & 0.237 \\
\hline & 3 & & & & & & 2 & & 0.412 & & 0.314 \\
\hline & 3 & & & & & cantá & 2 & 1.960 & 0.000 & 0.490 & 0.370 \\
\hline CallaRe097 & 3 & & 0.619 & 0.489 & & & 3 & 2.111 & 0.550 & & 0.431 \\
\hline CallaRe100 & 3 & 2.246 & 0.500 & 0.555 & 0.456 & & 2 & 1.498 & 0.316 & 0.332 & 0.277 \\
\hline CallaRe101 & 3 & 1.893 & 0.095 & 0.472 & 0.397 & CallaRe191 & 2 & 1.205 & 0.188 & 0.170 & 0.155 \\
\hline CallaRe106 & 4 & 2.431 & 0.684 & & & CallaRe194 & 2 & 1.600 & 0.500 & 0.375 & 0.305 \\
\hline CallaRe109 & 3 & 2.256 & 0.619 & 0.557 & 0.462 & CallaRe198 & 3 & 2.492 & 0.650 & 0.599 & 0.514 \\
\hline
\end{tabular}

419

420

421

422

423

424

425

426

427

428

429

430

431

432

433

434

435

Specific EST-SSR markers were used to assess the genetic diversity and relationships among the 21 accessions of colored calla lily from New Zealand, the Netherlands, the United States and China. Of these tested markers, 58 (or $75.3 \%$ ) were polymorphic, while the others were monomorphic. The polymorphic EST-SSRs consisted of 22 di-, 18 tri-, 6 tetra-, 4 penta-motif- and 8 compound-motif-based markers. The raw data and characteristics of the 58 polymorphic EST-SSR markers in 21 accessions are listed in Table S8 and Table 5. The number of alleles per marker $(\mathrm{Na})$ ranged from 2 to 6, with 174 alleles in total. The average effective number of alleles per locus $(\mathrm{Ne})$ was 2.163 , with a maximum of 4.762 and a minimum of 1.156 . The observed heterozygosity $(\mathrm{Ho})$ varied from 0 to 1.000 , whereas the expected heterozygosity $(\mathrm{He}$ ) varied from 0.135 to 0.790 . The mean $\mathrm{Ho}$ and $\mathrm{He}$ values were 0.483 and 0.491 , respectively. The polymorphic information content $(P I C)$ values ranged from 0.130 to 0.757 with a mean value of $0.420 . \mathrm{Nr}$ annotation results (Table S7) showed that most of the polymorphic SSR-containing sequences shared significant homology to protein-encoding genes from $V$. vinifera (Vitaceae), T. cacao (Sterculiaceae), Z. mays and O. sativa Indica (Gramineae), among others. These positive-hit homologous genes, including nucleic acid binding protein, 
436 glycine-rich protein, abscisic acid, zinc-finger transcription factor, had hypothetical or

437 putative functions in defense and stress, transporters and metabolic processes.
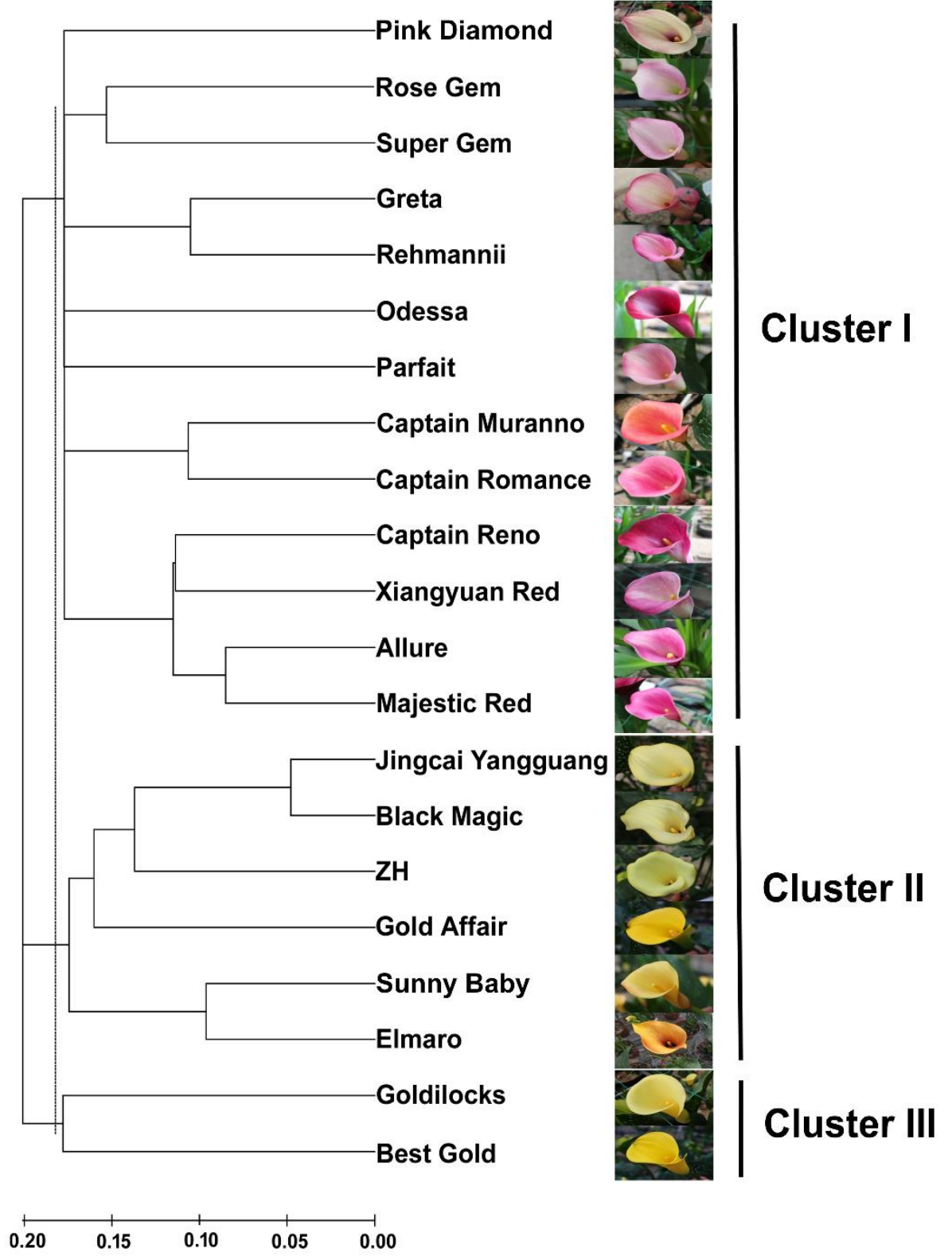

Figure 3 An NJ dendrogram of 21 colored calla lily accessions based on 58 polymorphic EST-SSR markers.

Based on the shared allele distance, we conducted a cluster analysis to assess the genetic relationships between 21 accessions of colored calla lily (Fig. 3). The dendrogram clearly grouped these accessions into three major clusters (I, II and III) at an approximate genetic distance of 0.18. Cluster I consisted of 13 accessions collected from the Netherlands, the United States, New Zealand and China. Twelve accessions in clusters I (Pink Diamond,

447 Rose Gem, Super Gem, Greta, Parfait, Rehmannii, Odessa, Majestic Red, Xiangyuan Red, 448 Captain Reno, Allure, and Captain Romance) had red, purple and pink spathes, whereas 449 Captain Murano had an orange spathe. Clusters II and III consisted of eight yellow-flower accessions. The accessions in the former cluster (Jingcai Yangguang, Black Magic, ZH, 
451 Gold affair, Sunny Baby and Elmaro) originated from New Zealand and China, while those

452 in the latter (Goldilocks and Butter Gold) were collected from New Zealand. The results

453 may indicate a potential association between the genetic relationship of the tested

454 accessions and the spathe color.

455

\section{DISCUSSION}

457 Illumina transcriptome sequencing and de novo assembly

458 Zantedeschia spp. are perennial bulb plants known worldwide for their ornamental uses.

459 These plants are even commonly used in traditional medicine in some African regions

460 (Letty, 1973; Singh, 1996). However, little genomic information is available for the plants

461 of the genus Zantedeschia, even with significant advances in DNA sequencing

462 technologies. The genomic researches of Zantedeschia spp. may be hindered by the large

463 genome size of these plants. The nuclear DNA contents of colored calla lily Z. elliottiana

464 and white calla lily Z. aethiopica (Ghimire et al., 2012) were estimated to be $1.17 \pm 0.50$

465 and $3.72 \pm 0.10 \mathrm{pg} \mathrm{DNA} / 2 \mathrm{C}$, respectively, equivalent to genome sizes of 1.15 and 3.64

466 Gbp, approximately 2.5 and 7.8 times larger than the rice (O. sativa) genome of $0.47 \mathrm{~Gb}$.

467 Moreover, Zantedeschia spp. may be a genetically high-heterozygosity bulb-flower

468 species. Since these species having been bred for more than one century, a larger number

469 of outstanding cultivars and hybrids of white and colored calla lilies in a range of sizes and

470 colors have been cross-bred, named and released by European and American breeders.

471 Thus, it is obviously not feasible to consider whole-genome sequencing for this perennial

472 plant because of the high cost and time consumption.

473 For organisms with large, heterozygous and complex genomes containing repetitive

474 sequences, RNA-seq-based de novo transcriptome analysis is an attractive alternative to

475 examine the properties of a transcriptome as a proxy for the whole genome (Martin \&

476 Wang, 2011; Onda et al., 2015). A transcriptome study of Z. aethiopica spathe (Cândido et

$477 a l ., 2014$ ) also demonstrated that transcriptome sequencing is a useful molecular biology

478 tool to rapidly build comprehensive sequence resources of expressed genes for in-depth

479 analysis at high resolution. To date, de novo transcriptome analysis has successfully been

480 applied to a wide range of plant species (Martin \& Wang, 2011), including crops, fruits,

481 vegetables, forest plants, flowers, and medicinal plants, for various purposes, such as

482 gaining fundamental insight into biological processes, generating different dynamic views

483 of interesting gene expression, discovering novel genes or transcripts, and developing and

484 validating molecular markers. In the present study, Illumina paired-end RNA-seq

485 technology was used to sequence the pooled RNA from ten tissues of 'Rehmannii'. More

486 than 59.9 million 101-bp paired-end reads were yielded, encompassing $6.1 \mathrm{G}$ nucleotides.

487 Trinity software (Haas et al., 2013) was then used to de novo assemble these short reads to

488 generate a total of 39,298 unigenes with an average length of 1,038 bp. The results

489 indicated that de novo transcriptome assembly is a feasible strategy to provide genome

490 information for non-model plant species. In sequence assembly, the N50 value is an

491 important statistical measure that is used to evaluate transcriptome assemblies in which a

492 high number corresponds to high quality. The N50 length of the 'Rehmannii' unigenes was 
$4931,476 \mathrm{bp}$, which is comparable to a recent report of the assembled transcriptome sequences

494 of $Z$. aethiopica (N50 = 1,600 bp) using a similar method (Cândido et al., 2014). However,

495 it should be noted that Z. aethiopica has approximately 1.5 times (91.2 million) as many

496 short reads as does 'Rehmannii'. These values are also larger than those reported for

497

498

499

500

501

502

503

504

505

506

507

508

509

510

511

512

513

514

515

516

517

518

519

520

521

522

523

524

525

526

527

528

529

530

531

532

533

534 related species in the Araceae family, such as A. andraeanum (N50 =1172 bp), A. konjac and $A$. bulbifer (N50 = 381 and 534; N50 = 372 and 524, respectively) (Zheng et al., 2013; Diao et al., 2014). Previous reports have demonstrated that an accurate and effective assembly tends to have a longer mean length and a larger N50 value (Chen et al., 2015), which suggests the high quality of our colored calla lily transcriptome sequences.

\section{Functional annotation and classification of unigenes}

To predict the biological functions of as many assembled transcripts as possible, various protein databases, including Nr, Swiss-Prot, COG, GO and KEGG, were employed. In total, 21,077 unigenes (Table S3) showed significant hits in the above five databases. The annotation rate of 'Rehmannii' unigenes was 53.6\%, which was higher than that of $Z$. aethiopica (39.7\%) using same method. The higher percentage in this study may be attributed to the higher frequency of unigenes longer than $500 \mathrm{bp}$ in the assembled transcripts. In general, the longer unigenes were more likely to have BLAST matches in the protein databases (Parchman et al., 2010). It was estimated that the percent of unigenes $>500$ bp in 'Rehmannii' and Z. aethiopica was $65.9 \%$ and $42.1 \%$, respectively. Novaes et al. (2008) reported that a high number of next-generation sequencing (NGS) short reads often cannot be matched to known genes because the significance of sequence similarity partially depends on the length of the query sequence. Therefore, the nonsignificantly annotated unigenes that are likely novel transcripts unique to Z. rehmannii 'Rehmannii' may be too short to allow for statistically meaningful matches.

A species-based distribution of the best hits from the BlastX search against the $\mathrm{Nr}$ database showed that $32.0 \%$ of the annotated sequences of 'Rehmannii' had similarity with dicotyledonous grape ( $V$. vinifera). This finding was confirmed by other transcriptome reports on Z. aethiopica (Cândido et al., 2014), A. andraeanum (Yang et al., 2015; Li et al., 2015), A. konjac and A. bulbifer (Zheng et al., 2013). In fact, calla lily and grape are a monocotyledonous herb and a dicotyledonous woody vine, respectively. So they are distant from each other genetically and evolutionarily. One possible reason for this perceived similarity is the absence of whole-genome sequences in public databases for any species of Araceae. These assembled transcriptome sequences may provide an important data resource for future studies on taxa-specific phenomena in the family Araceae. Consistent with previous studies (Cândido et al., 2014; Yang et al., 2015; Li et al., 2015; Zheng et al., 2013), the annotated unigenes were classified into 24 COG and 47 GO sub-terms or subcategories, indicating that our transcriptome data represented a broad diversity of transcripts in colored calla lily. In addition, a total of 4,532 unigenes were annotated and mapped to 117 KEGG pathways. Approximately $80 \%$ of top 20 hit pathways were involved in genetic information processing and metabolism, while the others were related to pathways involved in plant hormone signal transduction, plant-pathogen interaction, phagosome, and endocytosis (Fig. 2C). The most highly represented pathway was related 
535 to genetic information processing and metabolism, reflecting the fact that calla lily devotes

536 an enormous investment to gene transcription control and capacity, cell maintenance and

537

538

539

540

541

542

543

544

545

546

547

548

549

550

551

552

553

554

555

556

557

558

559

560

561

562

563

564

565

566

567

568

569

570

571

572

573

574

575

576

defense capacity (Cândido et al., 2014). Unigene annotation, together with the predicated

pathways, also facilitated the discovery of some key genes involved in flower development and function in colored calla lily. In total, we identified 117 homologous sequences involved in eight pathways, including the anthocyanin biosynthesis pathway, carotenoid biosynthesis pathway, photoperiod pathway, vernalization pathway, gibberellic acid pathway, autonomous or others. These captured unigenes again demonstrated that a relatively accurate and high-coverage genomic database can be produced by RNA-seqbased de novo transcriptome analysis for non-model plant species.

\section{Identification of EST-SSRarker frequency and type}

Markers based on expressed sequences are useful and attractive for the detection of functional variation and gene-based analysis. However, the available EST-SSR markers are insufficient for the genus Zantedeschia at the present time. Wei et al. (2012) identified 209 EST-SSRs from 2,175 non-redundant ESTs derived from cDNA libraries of developing spathe in Z. aethiopica. Among these, a total of 166 primer pairs flanking the EST-SSRs could be designed. Note here that these EST-SSRs were identified based on Sanger sequencing. Very limited expressed sequence data can be generally produced via the Sanger sequencing of cDNA libraries compared to RNA-based transcriptome sequencing. In our study, 9,933 potential EST-SSRs were identified in the de novo transcriptome sequences obtained by Illumina sequencing (Table 2). These data confirm that transcriptome sequences are excellent resources for the development of numerous SSR markers. In the present study, it was estimated that approximately $20.3 \%(7,997)$ of the assembled unigenes possess SSR loci, and the abundance of SSRs was one SSR locus per $4.1 \mathrm{~kb}$. The density of SSR-containing sequences in 'Rehmannii' was higher than that in the transcriptome reports for other species in Araceae, such as Amorphophallus spp. (11.8\%) and P. ternate (16.24\%) (Wang et al., 2014; Zheng et al., 2013). The frequency of SSRs was higher than that in P. ternate (4.3 kb) (Zheng et al., 2013) but lower than that in Amorphophallus spp. (3.6 kb) (Wang et al., 2014). The difference in the abundance estimation and frequency of SSRs among various species could partially be due to the SSR search criteria, the size of the unigene assembly dataset, the database-mining tools and the sequence redundancy, in addition to actual differences between species (Wang et al., 2014; Zheng et al., 2013; Chen et al., 2015).

The SSRs identified in this study were not uniformly distributed in the 'Rehmannii' transcriptome database (Table 3 ). When mononucleotide repeats were excluded, di$(59.8 \%)$ and trinucleotide repeats $(38.8 \%)$ were the most abundant, whereas hexa- $(1.1 \%)$, penta- $(0.2 \%)$ and tetranucleotide repeats $(0.1 \%)$ were rare. This result is identical to the previous findings of di- and trinucleotide motifs as the most frequent SSR motif types in the transcriptome sequences of many other plants, including Amorphophallus spp. and $P$. ternate (Wang et al., 2014; Zheng et al., 2013). As shown in Table 3, the AG/CT motif was the most abundant dinucleotide repeat (27.9\%), followed by AT/TA (3.6\%) and AC/GT (3.3\%). The predominant AG/CT motif repeats were also observed in Amorphophallus spp. 
577 and P. ternate (Wang et al., 2014; Zheng et al., 2013). The most abundant trinucleotide

578 repeat motif in calla lily was AGG/CCT, closely followed by AAG/CTT, similar to reports

579 in Amorphophallus spp. but different from those in P. ternate (Wang et al., 2014; Zheng et

$580 a l ., 2013)$. This difference possibly arises from the SSR search parameters and search

581 algorithms. CCG/CGG was the most frequent trinucleotide motif in $P$. ternate but the third

582 and fourth most common repeat type in Amorphophallus spp. and colored calla lily

583 'Rehmannii', respectively. Anyhow, these abundance results corroborated with the

584 suggestion that the trinucleotide motif CCG/CGG is common in monocots. In addition, we

585 also noticed that GC-rich trinucleotide motifs (ACC/GGT, ACG/CGT, AGC/CTG,

$586 \mathrm{AGG} / \mathrm{CCT}$ and CCG/CGG comprised $>65 \%$ ) were more abundant than AT-rich

587 trinucleotide motifs (AAG/CTT, AAT/ATT, ACT/AGT, and ATC/ATG comprised < 35\%)

588 in these three Araceae species. These results strongly support the fact that the high GC

589 content and consequent codon usage bias are specific features of monocot genomes

590 (Morgante, Hanafey \& Powell, 2002).

591 Evaluation of genetic diversity and relationships among colored calla lily accessions

592 Cultivar 'Rehmannii' in the present study is a hybrid of Z. rehmannii. It has the desired

593 attributes for potted flower purposes, such as pink spathes, perfect trumpet-shaped

594 inflorescence, lanceolate and semi-erect leaves, and high productivity. Similar

595 characteristics were also observed in another four cultivars, including Super Gem, Rose

596 Gem, Xiangyuan Red and Allure. Hence, all five varieties here were initially used to

597 evaluate EST-SSR marker usefulness in monitoring polymorphisms. A total of 200 primer

598 pairs were synthesized and tested, of which 137 (68.5\%) successfully yielded amplicons in

599 these five cultivars. Of the abovementioned working primer pairs, 77 (56.2\%) produced

600 PCR products of the expected fragment size used to screen for polymorphisms among 21

601 individual accessions. Finally, 58 polymorphic EST-SSR markers were obtained with a

602 polymorphic proportion of $75.3 \%$. EST-SSR markers are advantageous to SSRs in non-

603 transcribed regions due to their higher amplification rates and cross-species transferability

604 (Varshney, Graner \& Sorrells, 2005). The results here indicate that these transcript-based

605 SSRs are conserved in the germplasms of colored calla lily, suggesting that they will have

606 a broad utilization in taxonomic and cultivar identification, as well as

607 comparative mapping. The amplification and polymorphic rate of the EST-SSRs developed

608 in our study (59.3\%) is much higher than that obtained in Z. aethiopica using Sanger

609 sequencing (Wei et al., 2012), suggesting that the de novo transcriptome sequence based on

610 Illumina RNA-seq was accurate and of high quality. In Z. aethiopica (Wei et al., 2012), 68

611 (40.9\%) of the EST-SSR primer pairs yielded PCR amplification products in 24

612 accessions, of which 43 (63.2\%) exhibited polymorphisms. However, the result is lower

613 than that reported in Amorphophallus spp. (Zheng et al., 2013). In A. konjac and A.

614 bulbifer, $270(84.4 \%)$ primer pairs produced amplicons in two wild accessions, and 205

$615(89.1 \%)$ EST-SSR markers were polymorphic between 25 wild and cultivated accessions.

616 The low amplification and polymorphism rate in colored calla lily may be attributed to

617 various factors (Varshney et al., 2007; Varshney, Graner \& Sorrells, 2005), including the

618 presence of introns in the corresponding cDNA, SNPs or InDels (insertion-deletions) in the 


\section{Manuscript to be reviewed}

619 primers, assembly errors in the de novo transcriptome sequences, and the high

620

621

622

623

624

625

626

627

628

629

630

631

632

633

634

635

636

637

638

639

640

641

642

643

644

645

646

647

648

649

650

651

652

653

654

655

656

657

658

659

660 heterozygosity of the calla lily genome. Nonetheless, the failed amplification may be remedied if the PCR amplification conditions are re-optimized, such as by applying a lower annealing temperature and/or using gradient PCR.

Most calla lily cultivars have been bred following the intra/inter-specific hybridization of the species Z. rehmannii, Z. albomaculata, Z. elliottiana and Z. pentlandii within the section Aestivae, producing plants and flowers with a broad range of shapes and colors. The species Z. rehmannii, Z. albomaculata, Z. elliottiana and Z. pentlandii are closely related to each other based on cytogenetic karyotypes (Yao et al., 1994b). However, the genetic relationship among commercial hybrids or varieties is still not well defined. Characters based on which species or even varieties have been previously separated, for example, the degree of spotting on the leaf, the presence of bristles on the petioles and peduncles and, to some extent, the leaf shape, have been unreliable when a wide range of hybrids is examined (Letty, 1973; Singh, 1996). Molecular markers, such as RAPD and ISSR (Hamada \& Hagimori, 1996; Zhang et al., 2009; Lu et al., 2012; Chen et al., 2013; Lu et al., 2014), therefore, were used for cultivar identification and to evaluate the genetic diversity and relationship of germplasm resources of colored calla lily. However, very limited and uncertain information has been obtained until now. EST-SSR markers facilitate better cross-genome comparisons and genetic diversity and relationship evaluation because their target coding domains are more likely to be conserved between relatives (Morgante, Hanafey \& Powell, 2002; Varshney, Graner \& Sorrells, 2005). In the present study, 174 alleles were identified in 21 accessions using 58 polymorphic EST-SSR markers with an average of 3.0 alleles per locus. The discriminating power, as determined by the PIC value, ranged from 0.130 to 0.757 with a mean value of 0.420 . Wei et al. (2012) reported 43 ESTSSR markers with an average gene diversity of 0.446 (PIC) in 24 Z. aethiopica individual plants. The polymorphism level here was comparable to that of the EST-SSR based study in Z. aethiopica.

A cluster analysis based on 58 polymorphic EST-SSR markers was then used to assess the genetic relationships among 21 accessions. Three major groups were identified at a cutoff genetic distance index of 0.18 . Cluster I consisted of thirteen accessions with a complex set of colored spathes, including red, purple, and pink, among others. The cultivated species Z. rehmannii cv. Rehmannii and another four cultivars (Super Gem, Rose Gem, Xiangyuan Red and Allure) used in the initial validation of EST-SSR markers were grouped into several sub-clusters. These sub-clusters may reflect that $Z$. rehmannii is easily crossed with other species (Letty, 1973; Funnell, 1993; Singh, 1996), giving rise to hybrids with lobed leaves and spathes that vary in color and shape. Surprisingly, eight accessions with yellow flowers were separated into Clusters II and III. The cultivars Black Magic in Cluster II and Best Gold in Cluster III are intra-species hybrids of Z. elliottiana and Z. pentlandii, respectively, representing the close relationship between Clusters II and III, as well as Z. elliottiana and Z. pentlandii. The species Z. elliottiana and Z. pentlandii, also known as Yellow Arum (Letty, 1973; Singh, 1996), have leaves that are hastate to cordate at the base and differ in their consistency of yellow spathes. These species are distant from 
661 the other species Z. albomaculata and Z. rehmannii. Ronald et al. (2007) determined the

662 plastome composition of Z. aethiopica, Z. rehmannii, Z. albomaculata subsp.

663 albomaculata, Z. albomaculata subsp. macrocarpa, Z. elliottiana and Z. pentlandii with

664 species-specific CAPS markers developed from the plastidial intergenic region of $t r n \mathrm{D}$ and $665 \operatorname{trnC}(\mathrm{DC})$. They revealed that Z. elliottiana and Z. pentlandii showed a DC-AluI and a DC-

666 HaeIII restriction pattern that differed from that of $Z$. rehmannii and $Z$. albomaculata. The 667 results presented here also support the above findings and suggest that EST-SSR makers

668 developed from de novo transcriptome analysis are potential tools for taxonomy and 669 cultivar identification in the genus Zantedeschia. Interestingly, the genetic relationships

670 among colored calla lily accessions seemly related to the spathe color. However, we should note that this conclusion was drawn from limited numbers of Zantedeschia accessions in section Aestivae. A higher number of accessions and more individual cultivated plants will be essential for verifying the abovementioned relationship in future studies.

674

\section{CONCLUSIOINS}

676 This study was an attempt to present the transcriptome of colored calla lily using Illumina next-generation sequencing and de novo assembly. A total of 39,298 unigenes with an average length of 1,038 bp were generated, of which $53.6 \%(21,077)$ were annotated using the Nr, Swiss-Prot, COG, GO and KEGG databases. Based on the transcriptome dataset, we identified a total of 117 unique transcripts related to flower development, including pigment biosynthesis, floral organ development, flowering regulation and flower senescence. Moreover, a large number of SSRs and SNPs were mined and identified, and high-quality primers of 200 SSR loci were designed and demonstrated for their amplification and cross-species transferability in germplasm resources of colored calla lily. Finally, a relatively distinct genetic relationship among 21 accessions in section Aestivae was elucidated via 58 EST-SSR markers. The enrichment results highlight the potential of a de novo transcriptome dataset for functional genomics studies and molecular marker development. The EST-SSR markers generated in this study will enhance the current repository for the genus Zantedeschia and will be useful for taxonomic study and crop improvement programs.

691

\section{REFERENCES}

Ashburner M, Ball CA, Blake JA, Botstein D, Butler H, Cherry JM, Davis AP, Dolinski K, Dwight SS, Eppig JT, Harris MA, Hill DP, Issel-Tarver L, Kasarskis A, Lewis S, Matese JC, Richardson JE, Ringwald M, Rubin GM, Sherlock G. 2000. Gene ontology: tool for the unification of biology. Nature Genetics 25:25-29 DOI 10.1038/75556.

Cândido EDS, Fernandes GDR, Alencar SAD, Cardoso MHES, Lima SMDF, Miranda VDJ, Porto WF, Nolasco DO, Oliveira-Júnior NGD, Barbosa AEAD, Pogue RE, Rezende TMB, Dias SC, Franco OL. 2014. Shedding some light over the floral metabolism by arum lily (Zantedeschia aethiopica) spathe de novo transcriptome assembly. PLoS ONE 9:e90487 DOI 10.1371/journal.pone.0090487. 


\section{Manuscript to be reviewed}

703 Chen H, Liu L, Wang L, Wang S, Somta P, Cheng X. 2015. Development and

704

705

706

707

708

709

710

711

712

713

714

715

716

717

718

719

720

721

722

723

724

725

726

727

728

729

730

731

732

733

734

735

736

737

738

739

740

741

742

743

744

745

746

Validation of EST-SSR Markers from the Transcriptome of Adzuki Bean (Vigna angularis). PLoS ONE 10:e131939 DOI 10.1371/journal.pone.0131939.

Chen Z, Xu BL, Pu CJ, Xu Q, Tian G. 2013. Physiological and Biochemical and resistance changes and ISSR polymorphic analysis exposed to ${ }^{12} \mathrm{C}^{6+}$ heavy ion radiation on calla lily. Journal of Nuclear Agricultural Sciences 27:0552-0556 DOI 10.11869/hnxb.2013.05.0552.

Diao Y, Yang CZ, Yan M, Zheng XF, Jin SR, Wang YW, Hu ZL. 2014. De novo transcriptome and small RNA analyses of two Amorphophallus species. PLoS One 9: e95428 DOI org/10.1371/journal.pone.0095428.

Funnell KA, MacKay BR. 1999. Directions and challenges of the New Zealand calla industry, and the use of calcium to control soft rot. In International Symposium on Development of Bulbous Flower Industry; Sheen TF, Chen JJ, Yang TC, Eds.; Taiwan Seed Improvement and Propagation Station: Taichung, Taiwan, 30-31.

Funnell KA. Zantedeschia. 1993. In The physiology of flower bulbs. Hertogh AD, Nard ML, Eds.; Elsevier: Amsterdam, Netherlands, 683-703.

Ghimire BK, Yu CY, Kim HJ, Chung IM. 2012. Karyotype and nucleic acid content in Zantedeschia aethiopica Spr. and Zantedeschia elliottiana Engl.. African Journal of Biotechnology 11:11604-11609 DOI 10.5897//AJB12.06.

Haas BJ, Papanicolaou A, Yassour M, Grabherr M, Blood PD, Bowden J, Couger MB, Eccles D, Li B, Lieber M, MacManes MD, Ott M, Orvis J, Pochet N, Strozzi F, Weeks N, Westerman R, William T, Dewey CN, Henschel R, Leduc RD, Friedman N, Regev A. 2013. De novo transcript sequence reconstruction from RNAseq using the Trinity platform for reference generation and analysis. Nature Protocols 8:1494-1512 DOI 10.1038/nprot.2013.084.

Hamada K, Hagimori M. 1996. RAPD-based method for cultivar-identification of calla lily (Zantedeschia spp.). Scientia Horticulturae 65:215-218 DOI 10.1016/03044238(95)00869-1.

Kanehisa M, Goto S, Kawashima S, Okuno Y, Hattori M. 2004. The KEGG resource for deciphering the genome. Nucleic Acids Research 32:277-280.

Letty C. 1973. The genus Zantedeschia. Bothalia 11:5-26 DOI 10.4102/abc.v11i1\&2.1963.

Li Z, Wang JB, Zhang XQ, Xu L. 2015. Comparative transcriptome analysis of Anthurium "Albama" and its anthocyanin-loss mutant. PLoS One 10:e0119027 DOI 10.1371/journal.pone.0119027.

Liu K, Muse SV. 2005. PowerMarker: an integrated analysis environment for genetic marker analysis. Bioinformatics 21:2128-2129.

Lu B, Zheng YH, Peng F, Shu XC, Chen XX. 2012. Optimization of RAPD reaction system by uniform design on Zantedeschica hybrid. Northern Horticulture 11:123126.

Lu Bo, Zheng YH, Chen M, Peng F, Shu XC, Gao F. 2014. Effects of 60 Co- $\gamma$ ray radiation on color Lily Parfait and their physiological responses to high temperature and humidity. Journal of Nuclear Agricultural Sciences 28:1353-1357 DOI 10.11869/j.issn.100-8551.2014.08.1353. 
Martin JA, Wang Z. 2011. Next-generation transcriptome assembly. Nature Reviews Genetics 12:671-682 DOI 10.1038/nrg3068.

Morgante M, Hanafey M, Powell W. 2002. Microsatellites are preferentially associated with non-repetitive DNA in plant genomes. Nature Genetic 30:194-200.

Novaes E, Drost D, Farmerie WG, Pappas GJ, Grattapaglia D, Ronald R, Kirst M. 2008. High-throughput gene and SNP discovery in Eucalyptus grandis, an uncharacterized genome. BMC Genomics 9:312 DOI 10.1186/1471-2164-9-312.

Onda Y, Mochida K, Yoshida T, Sakurai T, Seymour RS, Umekawa Y, Pirintsos SA, Shinozaki K. 2015. Transcriptome analysis of thermogenic Arum concinnatum reveals the molecular components of floral scent production. Scientific Reports 5:8753 DOI 10.1038/srep08753.

Parchman TL, Geist KS, Grahnen JA, Benkman CW, Buerkle CA. 2010. Transcriptome sequencing in an ecologically important tree species: assembly, annotation, and marker discovery. BMC Genomics 11:180 DOI 10.1186/1471-216411-180.

Peakall R, Smous PE. 2006. GenAlEx 6: Genetic analysis in Excel. Population genetic software for teaching and research. Molecular Ecology Notes 6:288-295 DOI 10.1111/j.1471-8286.2005.01155.x.

Roman LT, Michael YG, Darren AN, Eugene VK. 2000. The COG database: a tool for genome-scale analysis of protein functions and evolution. Nucleic Acids Research 28:33-36.

Singh Y. 1996. Contributions to the systematic of the genus Zantedeschia Spreng.( Araceae); University of Pretoria Press: Pretoria, South Africa, 169.

Snijder RC, Santiago BF, Tuyl VJM. 2007. The role of plastome-genome incompatibility and biparental plastid inheritance in interspecific hybridization in the genus Zantedeschia (Araceae). Floriculture and Ornamental Biotechnology 1:150157 DOI 10.1007/BF00225906.

Tamura K, Peterson D, Peterson N, Stecher G, Nei M, Kumar S. 2011. MEGA5: Molecular evolutionary genetic analysis using maximum likelihood, evolutionary distance, and maximum parsimony methods. Molecular Biology and Evolution 28:2731-2739 DOI 10.1093/molbev/msr121.

Tian DQ, Pan XY, Yu YM, Wang WY, Zhang F, Ge YY, Shen XL, Shen FQ, Liu XJ. 2013. De novo characterization of the Anthurium transcriptome and analysis of its digital gene expression under cold stress. BMC Genomics 14:1-14 DOI 10.1186/1471-2164-14-827.

Tjia B. 1985. Hybrid calla lilies: A potential new crop for Florida. Proceedings of the Florida State Horticultural Society 98:127-130.

Varshney RK, Graner A, Sorrells ME. 2005. Genic microsatellite markers in plants: features and applications. Trends in Biotechnology 23:48-55.

Varshney RK, Mahendar T, Aggarwal RK, Börner A. 2007. Genic molecular markers in plants: development and applications. In Genomics-assisted crop improvement; Varshney, R.K., Tuberosa, R. Eds.; Springer Netherlands: Belgium, Germany, 13-29.

Wang S, Zhang Z, Jiang NH, Zhang GH, Sha BC, Yang SC, Chen JW. 2014. SSR information in transcriptome of Pinellia ternata. Zhong Yao Cai 37:1566-1569 DOI 10.13863/j. issn1001-4454.2014.09.015.

Wei ZZ, Luo LB, Zhang HL, Xiong M, Wang X, Zhou D. 2012. Identification and characterization of 43 novel polymorphic EST-SSR markers for arum lily, Zantedeschia aethiopica (Araceae). American Journal of Botany 99:e493-497 DOI 10.3732/ajb.1200228. 


\section{Manuscript to be reviewed}

796 Yang YX, Chen XX, Xu B, Li YX, Ma YH, Wang GD. 2015. Phenotype and transcriptome analysis reveals chloroplast development and pigment biosynthesis together influenced the leaf color formation in mutants of Anthurium andraeanum 'Sonate'. Frontiers in Plant Science 6:139 DOI 10.3389/fpls.2015.00139.

Yao JL, Cohen D, Rowland RE. 1994. Karyotype studies in the genus Zantedeschia.

802 South African Journal of Botany 60:4-7.

803

804

Yao JL, Cohen D, Rowland RE. 1995. Interspecific albino and variegated hybrids in the genus Zantedeschia. Plant Science 109:199-206 DOI 10.1016/0168-9452(95) 04163$\mathrm{O}$.

Yao JL, Cohen D, Rowland RE. 1994. Plastid DNA inheritance and plastome-genome incompatibility in interspecific hybrids of Zantedeschia (Araceae). Theoretical and Applied Genetics 88:255-260 DOI 10.1007/BF00225906.

Zhang YC, Tang GG, Chu YX, Wu AZ. 2009. Optimization of ISSR reaction system and preliminary study on Zantedeschia. Molecular Plant Breeding 7:827-832 DOI 10.3969/mpb.007.000827.

Zheng XF, Pan C, DiaoY, You YN, Yang CZ, Hu ZL. 2013. Development of microsatellite markers by transcriptome sequencing in two species of Amorphophallus (Araceae). BMC Genomics 14:490 DOI 10.1186/1471-2164-14-490. 